\title{
Riemannian submersions from compact four manifolds
}

\author{
Xiaoyang Chen *
}

\begin{abstract}
We show that under certain conditions, a nontrivial Riemannian submersion from positively curved four manifolds does not exist. This gives a partial answer to a conjecture due to Fred Wilhelm. We also prove a rigidity theorem for Riemannian submersions with totally geodesic fibers from compact four-dimensional Einstein manifolds.
\end{abstract}

\section{Introduction}

A smooth map $\pi:(M, g) \rightarrow(N, h)$ is a Riemannian submersion if $\pi_{*}$ is surjective and satisfies the following property:

$$
g_{p}(v, w)=h_{\pi(p)}\left(\pi_{*} v, \pi_{*} w\right)
$$

for any $v, w$ that are tangent vectors in $T M_{p}$ and perpendicular to the kernel of $\pi_{*}$.

A fundamental problem in Riemannian geometry is to study the interaction between curvature and topology. A lot of important work has been done in this direction. In this paper we study a similar problem for Riemannian submersions:

Problem: Explore the structure of $\pi$ under additional curvature assumptions of $(M, g)$.

When $(M, g)$ has constant sectional curvature, we have the following classification results $(8],[21],[22])$.

Theorem 1.1. Let $\pi:\left(M^{m}, g\right) \rightarrow(N, h)$ be a nontrivial Riemannian submersion (i.e. $0<$ $\operatorname{dim} N<\operatorname{dim} M)$ with connected fibers, where $\left(M^{m}, g\right)$ is compact and has constant sectional curvature $c$.

1. If $c<0$, then there is no such Riemannian submersion.

2. If $c=0$, then locally $\pi$ is the projection of a metric product onto one of its factors.

3. If $c>0$ and $M^{m}$ is simply connected, then $\pi$ is metrically congruent to the Hopf fibration, i.e, there exist isometries $f_{1}: M^{m} \rightarrow \mathbb{S}^{m}$ and $f_{2}: N \rightarrow \mathbb{P}(\mathbb{K})$ such that $p f_{1}=f_{2} \pi$, where $p$ is the standard projection from $\mathbb{S}^{m}$ to projective spaces $\mathbb{P}(\mathbb{K})$.

However, very little is known about the structure of $\pi$ if $(M, g)$ is not of constant curvature. In this paper we consider two different curvature conditions:

1. $(M, g)$ has positive sectional curvature.

\footnotetext{
${ }^{*}$ The author is supported in part by NSF DMS-1209387.
} 
2. $(M, g)$ is an Einstein manifold.

When $(M, g)$ has positive sectional curvature, we have the following important conjecture due to Fred Wilhelm (although never published anywhere).

Conjecture 1 Let $\pi:(M, g) \rightarrow(N, h)$ be a nontrivial Riemannian submersion, where $(M, g)$ is a compact Riemannian manifold with positive sectional curvature. Then $\operatorname{dim}(F)<\operatorname{dim}(N)$, where $F$ is the fiber of $\pi$.

By Frankel's theorem [7], it is not hard to see that Conjecture 1 is true if at least two fibers of $\pi$ are totally geodesic. In fact, since any two fibers do not intersect with each other, Frankel's theorem implies that $2 \operatorname{dim}(F)<\operatorname{dim}(M)$. Hence $\operatorname{dim}(F)<\operatorname{dim}(N)$. If all fibers of $\pi$ are totally geodesic, we have the following much stronger result:

Proposition 1.2. Let $\pi:(M, g) \rightarrow(N, h)$ be a nontrivial Riemannian submersion such that all fibers of $\pi$ are totally geodesic, where $(M, g)$ is a compact Riemannian manifold with positive sectional curvature. Then $\operatorname{dim}(F)<\rho(\operatorname{dim}(N))+1$, where $F$ is any fiber of $\pi$ and $\rho(n)$ is the maximal number of linearly independent vector fields on $S^{n-1}$.

Notice that we always have $\rho(\operatorname{dim}(N))+1 \leq \operatorname{dim}(N)-1+1=\operatorname{dim}(N)$ and equality holds if and only $\operatorname{dim}(N)=2,4$ or 8 .

Although not explicitly stated, Proposition 1.2 appears in [6]. For completeness, we will give a proof in section 3 .

When $\operatorname{dim}(M)=4$, Conjecture 1 is equivalent to the following conjecture.

Conjecture 2 There is no nontrivial Riemannian submersion from any compact four manifold $\left(M^{4}, g\right)$ with positive sectional curvature.

In fact, suppose there exists such a Riemannian submersion $\pi:\left(M^{4}, g\right) \rightarrow(N, h)$. Then Conjecture 1 would imply $\operatorname{dim}(N)=3$. Hence the Euler number of $M^{4}$ is zero. On the other hand, since $\left(M^{4}, g\right)$ has positive sectional curvature, $H^{1}\left(M^{4}, \mathbb{R}\right)=0$ by Bochner's vanishing theorem ([15], page 208). By Poincaré duality, the Euler number of $M^{4}$ is positive. Contradiction.

Let $\pi:(M, g) \rightarrow(N, h)$ be a Riemannian submersion. We say that a function $f$ defined on $M$ is basic if $f$ is constant along each fiber. A vector field $X$ on $M$ is basic if it is horizontal and is $\pi$-related to a vector field on $N$. In other words, $X$ is the horizontal lift of some vector field on $N$. Let $H$ be the mean curvature vector field of the fibers and $A$ be the O'Neill tensor of $\pi$. We denote by $\|A\|$ the norm of $A$, i.e., $\|A\|^{2}=\sum_{i, j}\left\|A_{X_{i}} X_{j}\right\|^{2}$, where $\left\{X_{i}\right\}$ is a local orthonormal basis of the horizontal distribution of $\pi$. The next theorem gives a partial answer to Conjecture 2 .

Theorem 1.3. There is no nontrivial Riemannian submersion from any compact four manifold with positive sectional curvature such that either $\|A\|$ or $H$ is basic.

We emphasize that in Conjecture 1 the assumption that $(M, g)$ has positive sectional curvature can not be replaced by $(M, g)$ has positive sectional curvature almost everywhere, namely, $(M, g)$ has nonnegative sectional curvature everywhere and has positive sectional curvature on an open and dense subset of $M$. Indeed, Let $g$ be the metric on $S^{2} \times S^{3}$ constructed by B. Wilking which has positive sectional curvature almost everywhere [23. Then by a theorem 
of K. Tapp [18], $g$ can be extended to a nonnegatively curved metric $\tilde{g}$ on $S^{2} \times \mathbb{R}^{4}$ such that $\left(S^{2} \times S^{3}, g\right)$ becomes the distance sphere of radius 1 about the soul. By Proposition 5.1, we get a Riemannian submersion $\pi:\left(S^{2} \times S^{3}, g\right) \rightarrow\left(S^{2}, h\right)$, where $h$ is the induced metric on the soul $S^{2}$ from $\tilde{g}$. This example shows that in Conjecture 1 the assumption that $(M, g)$ has positive sectional curvature can not be replaced by $(M, g)$ has positive sectional curvature almost everywhere.

Riemannian submersions are also important in the study of compact Einstein manifolds, for example, see [3]. Our next theorem gives a complete classification of Riemannian submersions with totally geodesic fibers from compact four-dimensional Einstein manifolds.

Theorem 1.4. Suppose $\pi:\left(M^{4}, g\right) \rightarrow(N, h)$ is a Riemannian submersion, where $\left(M^{4}, g\right)$ is a compact four-dimensional Einstein manifold. If all fibers of $\pi$ are totaly geodesic and have dimension 2 , then locally $\pi$ is the projection of a metric product $B^{2}(c) \times B^{2}(c)$ onto one of the factors, where $B^{2}(c)$ is a two-dimensional compact manifold with constant curvature $c$.

If the dimension of the fibers of $\pi$ is 1 or 3 (all fibers are not necessarily totally geodesic), then the Euler number of $M^{4}$ is zero. By a theorem of Berger [2, 13], $\left(M^{4}, g\right)$ must be flat. Hence by a theorem of Walschap [21, locally $\pi$ is the projection of a metric product $B^{2}(c) \times B^{2}(c)$ onto one of the factors.

Acknowledgment This paper is a part of my Ph.D thesis at University of Notre Dame [5]. The author would like to express gratitude to his advisor, Professor Karsten Grove, for many helpful discussions. He also thanks Professor Anton Petrunin for discussing the proof of Theorem 3.1 . The author benefits a lot from his "Exercises in orthodox geometry" [16].

\section{Preliminaries}

In this section we recall some definitions and facts on Riemannian submersions which will be used in this paper. We refer to [14] for more details.

Let $\pi:(M, g) \rightarrow(N, h)$ be a Riemannian submersion. Then $\pi$ induces an orthogonal splitting $T M=\mathcal{H} \oplus \mathcal{V}$, where $\mathcal{V}$ is tangent to the fibers and $\mathcal{H}$ is the orthogonal complement of $\mathcal{V}$. We write $Z=Z^{h}+Z^{v}$ for the corresponding decomposition of $Z \in T M$. The O'Neill tensor $A$ is given by

$$
A_{X} Y=\left(\nabla_{X} Y\right)^{v}=\frac{1}{2}([X, Y])^{v}
$$

where $X, Y \in \mathcal{H}$ and are $\pi$-related to some vector field on $N$, respectively.

Fix $X \in \mathcal{H}$, define $A_{X}^{*}$ by

$$
\begin{gathered}
A_{X}^{*}: \mathcal{V} \rightarrow \mathcal{H} \\
V \mapsto-\left(\nabla_{X} V\right)^{h} .
\end{gathered}
$$

Then $A_{X}^{*}$ is the dual of $A_{X}$.

Define the mean curvature vector field $H$ of $\pi$ by

$$
H=\sum_{i}\left(\nabla_{V_{i}} V_{i}\right)^{h}
$$


where $\left\{V_{i}\right\}_{i=1}^{k}$ is any orthonormal basis of $\mathcal{V}$ and $k=\operatorname{dim} \mathcal{V}$.

Define the mean curvature form $\omega$ of $\pi$ by

$$
\omega(Z)=g(H, Z)
$$

where $Z \in T M$. It is clear that $i_{V} \omega=\omega(V)=0$ for any $V \in \mathcal{V}$.

We say that a function $f$ defined on $M$ is basic if $f$ is constant along each fiber. A vector field $X$ on $M$ is basic if it is horizontal and is $\pi$-related to a vector field on $N$. In other words, $X$ is the horizontal lift of some vector field on $N$. A differential form $\alpha$ on $M$ is called to be basic if and only $i_{V} \alpha=0$ and $\mathcal{L}_{V} \alpha=0$ for any $V \in \mathcal{V}$, where $\mathcal{L}_{V} \alpha$ is the Lie derivative of $\alpha$.

The set of basic forms of $M$, denoted by $\Omega_{b}(M)$, constitutes a subcomplex

$$
d: \Omega_{b}^{r}(M) \rightarrow \Omega_{b}^{r+1}(M)
$$

of the De Rham complex $\Omega(M)$. The basic cohomology of $M$, denoted by $H_{b}^{*}(M)$, is defined to be the cohomology of $\left(\Omega_{b}(M), d\right)$.

Proposition 2.1. The inclusion map $i: \Omega_{b}(M) \rightarrow \Omega(M)$ induces an injective map

$$
H_{b}^{1}(M) \rightarrow H_{D R}^{1}(M) .
$$

Proof. See pages $33-34$, Proposition 4.1 in [20].

\section{Proof of Proposition 1.2 and Theorem 1.3}

We first give a proof of Proposition 1.2 .

Proof. Fix $p \in M$ and choose $X_{p}$ to be any point in the unit sphere of $\mathcal{H}_{p}$. Extend $X_{p}$ to be a unit basic vector field $X$. Since all fibers of $\pi$ are totally geodesic, by O'Neill's formula ([14]), $K(X, V)=\left\|A_{X}^{*} V\right\|^{2}$ for any unit vertical vector field $V$. Since $K(X, V)>0$ by assumption, we see that $A_{X}^{*} V \neq 0$ for any $V \neq 0$. Let $v_{1}, v_{2}, \cdots v_{k}$ be any orthonormal basis of $\mathcal{V}_{p}$, where $k=\operatorname{dim}\left(F_{p}\right)$ and $F_{p}$ is the fiber passing through $p$. Then $A_{X}^{*}\left(v_{1}\right), A_{X}^{*}\left(v_{2}\right), \cdots A_{X}^{*}\left(v_{k}\right)$ are linearly independent and are perpendicular to $X_{p}$. Since $X_{p}$ could be any point in the unit sphere of $\mathcal{H}_{p}$, then we get $k$ linearly independent vector fields on the unit sphere of $\mathcal{H}_{p}$. By the definition of $\rho(\operatorname{dim} N)$, we see that $\operatorname{dim}\left(F_{p}\right)=k \leq \rho(\operatorname{dim}(N))<\rho(\operatorname{dim}(N))+1$.

Remark 1. It would be very interesting to know whether one can replace $\operatorname{dim}(F)<\operatorname{dim}(N)$ by $\operatorname{dim}(F)<\rho(\operatorname{dim}(N))+1$ in Conjecture 1. It would be the Riemannian analogue of Toponogov's Conjecture (page 1727 in [17]) and would imply that $\operatorname{dim}(N)$ must be even (In fact, if $\operatorname{dim}(N)$ is odd, then $\rho(\operatorname{dim}(N))=0$. Hence $\operatorname{dim}(F)<\rho(\operatorname{dim}(N))+1$ implies $\operatorname{dim}(F)=0$ and hence $\pi$ is trivial, contradiction). In particular, there would be no Riemannian submersion with onedimensional fibers from even-dimensional manifolds with positive sectional curvature.

Let $\left(M^{m}, g\right)$ be an $m$-dimensional compact manifold with positive sectional curvature, $m \geq 4$ and $\left(N^{2}, h\right)$ be a 2-dimensional compact Riemannian manifold. Now we are going to prove the following theorem which implies Theorem 1.3 . 
Theorem 3.1. There is no Riemannian submersion $\pi:\left(M^{m}, g\right) \rightarrow\left(N^{2}, h\right)$ such that

1. the Euler numbers of the fibers are nonzero and

2. either $\|A\|$ or $H$ is basic.

Remark 2. If Conjecture 1 is true, then there would be no Riemannian submersion $\pi:\left(M^{m}, g\right) \rightarrow$ $\left(N^{2}, h\right)$, where $\left(M^{m}, g\right)$ has positive sectional curvature and $m \geq 4$.

Before we prove Theorem 3.1, we firstly show how to derive Theorem 1.3. The proof is by contradiction. Suppose there exists a nontrivial Riemannian submersion $\pi:\left(M^{4}, g\right) \rightarrow(N, h)$ such that either $\|A\|$ or $H$ is basic, where $\left(M^{4}, g\right)$ is a compact four manifold with positive sectional curvature. Since $\left(M^{4}, g\right)$ has positive sectional curvature, $H^{1}\left(M^{4}, \mathbb{R}\right)=0$ by Bochner's vanishing theorem ([15], page 208). By Poincaré duality, $\chi\left(M^{4}\right)=2+b_{2}\left(M^{4}\right)$ is positive. By a theorem of Hermann [12], $\pi$ is a locally trivial fibration. Then $\chi\left(M^{4}\right)=\chi(N) \chi(F)$, where $F$ is any fiber of $\pi$. It follows that $\operatorname{dim}(N)=2$ and $\chi(F)$ is nonzero (hence all fibers have nonzero Euler numbers), which is a contradiction by Theorem 3.1 .

The proof of Theorem 3.1 is again by contradiction. Suppose $\pi:\left(M^{m}, g\right) \rightarrow\left(N^{2}, h\right)$ be a Riemannian submersion satisfying the conditions in Theorem 3.1, By passing to its oriented double cover, we can assume that $N^{2}$ is oriented. The idea of the proof of Theorem 3.1 is to construct a nowhere vanishing vector field (or line field) on some fiber of $\pi$, which will imply the Euler numbers of the fibers are zero. Contradiction.

Since $(M, g)$ has positive sectional curvature, by a theorem of Walschap [21], $\|A\|$ can not be identical to zero on $M$. Hence there exists $p \in M$ such that $\|A\|(p) \neq 0$.

If $\|A\|$ is basic, then $\|A\| \neq 0$ at any point on $F_{p}$, where $F_{p}$ is the fiber at $p$. Let $X, Y$ be any orthonormal oriented basic vector fields in some open neighborhood of $F_{p}$. Then $\left\|A_{X} Y\right\|^{2}=$ $\frac{1}{2}\|A\|^{2} \neq 0$ at any point on $F_{p}$. Define a map $s$ by

$$
\begin{gathered}
s: F_{p} \rightarrow T F_{p} \\
x \mapsto \frac{A_{X} Y}{\left\|A_{X} Y\right\|}(x) .
\end{gathered}
$$

Let $Z, W$ be another orthonormal oriented basic vector fields. Then $Z=a X+b Y$ and $W=$ $c X+d Y, a d-b c>0$. Then

$$
A_{Z} W=(a d-b c) A_{X} Y .
$$

Hence $s$ does not depend on the choice of $X, Y$. Then $s$ is a nowhere vanishing vector field on $F_{p}$. Thus the Euler number of $F_{p}$ is zero. Contradiction.

If $H$ is basic, the construction of such nowhere vanishing vector field (or line field) is much more complicated. Under the assumption that $H$ is basic, we firstly construct a metric $\hat{g}$ on $M^{m}$ such that $\pi:\left(M^{m}, \hat{g}\right) \rightarrow\left(N^{2}, h\right)$ is still a Riemannian submersion and all fibers are minimal submanifolds with respect to $\hat{g}$. Of course, in general $\hat{g}$ can not have positive sectional curvature everywhere. However, the crucial point is that there exists some fiber $F_{0}$ such that $\hat{g}$ has positive sectional curvature at all points on $F_{0}$. Pick any fiber $F_{1}$ which is close enough to $F_{0}$. Then using the classical variational argument, we construct a continuous codimension one distribution on $F_{1}$. Thus the Euler number of $F_{1}$ is zero. Contradiction. 
Now we are going to explain the proof of Theorem 3.1 in details. We firstly need the following lemmas:

Lemma 3.2. Suppose $\omega$ is the mean curvature form of a Riemannian submersion from compact Riemannian manifolds. If $\omega$ is a basic form, then it is a closed form.

Proof. See page 82 in [20] for a proof.

Lemma 3.3. Suppose $\pi:\left(M^{m}, g\right) \rightarrow(N, h)$ is a Riemannian submersion such that $H$ is basic, where $\left(M^{m}, g\right)$ is a compact Riemannian manifold with positive sectional curvature. Then there exists a metric $\hat{g}$ on $M^{m}$ such that $\pi:\left(M^{m}, \hat{g}\right) \rightarrow(N, h)$ is still a Riemannian submersion and all fibers are minimal submanifolds with respect to $\hat{g}$. Furthermore, there exists some fiber $F_{0}$ such that $\hat{g}$ has positive sectional curvature at all points on $F_{0}$.

Proof. The idea is to use partial conformal change of metrics along the fibers, see also page 82 in [20]. Let $\omega$ be the mean curvature form of $\pi$. Since $H$ is basic, $\omega$ is a basic form. Then $\omega$ is closed by Lemma 3.2. So $[\omega]$ defines a cohomological class in $H_{b}^{1}\left(M^{m}\right)$. Because $\left(M^{m}, g\right)$ has positive sectional curvature, $H_{D R}^{1}\left(M^{m}\right)=0$ by Bochner's vanishing theorem ([15], page 208). By Proposition 2.1, we see that $H_{b}^{1}\left(M^{m}\right)=0$. Then there exists a basic function $f$ globally defined on $M^{m}$ such that $\omega=d f$. Define $\hat{f}=f-\max _{p \in M^{m}} f(p)$. Then $\max _{p \in M^{m}} \hat{f}(p)=0$ and $\omega=d \hat{f}$. Let $\lambda=e^{\hat{f}}$ and define

$$
\hat{g}=\left(\lambda^{\frac{2}{k}} g_{v}\right) \oplus g_{h},
$$

where $k=\operatorname{dim}\left(M^{m}\right)-\operatorname{dim}(N), g_{v} / g_{h}$ are the vertical / horizontal components of $g$, respectively.

Since the horizontal components of $g$ remains unchanged, $\pi:\left(M^{m}, \hat{g}\right) \rightarrow(N, h)$ is still a Riemannian submersion. Now the mean curvature form $\hat{\omega}$ associated to $\hat{g}$ is computed to be

$$
\hat{\omega}=\omega-d \log \lambda=0 .
$$

Hence all fibers of $\pi$ are minimal submanifolds with respect to $\hat{g}$.

Let $\phi(p)=\lambda^{\frac{2}{k}}(p), p \in M^{m}$. Then

$$
\hat{g}=\left(\phi g_{v}\right) \oplus g_{h}
$$

Note for any $p \in M^{m}, 0<\phi(p) \leq 1$. Moreover, we have $\max _{p \in M^{m}} \phi(p)=1$. Let $p_{0} \in M^{m}$ such that $\phi\left(p_{0}\right)=1$ and $F_{0}$ be the fiber of $\pi$ passing through $p_{0}$. Since $f$ is a basic function on $M^{m}$, $\phi$ is also basic. Then $\phi \equiv 1$ on $F_{0}$, which will play a crucial role in our argument below. Of course, in general $\hat{g}$ can not have positive sectional curvature everywhere. However, by Lemma 3.4 below, we see that $\hat{g}$ still has positive sectional curvature at all points on $F_{0}$. (The reader should compare it to the following fact: Let $\hat{h}=e^{2 f} h$ be a conformal change of $h$, where $h$ is a Riemannian metric on $M$ with positive sectional curvature. Then $\hat{h}$ still has positive sectional curvature at those points where $f$ attains its maximum value.)

Indeed, by Lemma 3.4 below, for any basic vector fields $X, Y$ and vertical vector fields $V, W$, we have

$$
\begin{aligned}
\hat{K}(X & +V, Y+W)\|(X+V) \wedge(Y+W)\|^{2}=\hat{R}(X+V, Y+W, Y+W, X+V) \\
& =R(X+V, Y+W, Y+W, X+V)+(\phi-1) P(\nabla \phi, \phi, X, Y, V, W)
\end{aligned}
$$




$$
\begin{gathered}
+Q(\nabla \phi, \phi, X, Y, V, W)+\left[-g(W, W) g\left(\nabla_{V} \nabla \phi, X\right)\right. \\
+g(V, W) g\left(\nabla_{W} \nabla \phi, X\right)+g(V, W) g\left(\nabla_{V} \nabla \phi, Y\right) \\
\left.-g(V, V) g\left(\nabla_{W} \nabla \phi, Y\right)\right]+\frac{1}{2}[-H e s s(\phi)(X, X) g(W, W) \\
+2 \operatorname{Hess}(\phi)(X, Y) g(V, W)-\operatorname{Hess}(\phi)(Y, Y) g(V, V)],
\end{gathered}
$$

where $\hat{K}(X+V, Y+W)$ is the sectional curvature of the plane spanned by $X+V, Y+W$ with respect to $\hat{g}$ and

$$
\|(X+V) \wedge(Y+W)\|^{2}=\hat{g}(X+V, X+V) \hat{g}(Y+W, Y+W)-[\hat{g}(X+V, Y+W)]^{2} .
$$

Moreover, $\nabla$ is the Levi-Civita connection and $\operatorname{Hess}(\phi)$ is the Hessian of $\phi$ with respect to $g$. Also $\hat{R} / R$ are the Riemannian curvature tensors with respect to $\hat{g} / g$, respectively. Furthermore, $P(\nabla \phi, \phi, X, Y, V, W), Q(\nabla \phi, \phi, X, Y, V, W)$ are two functions depending on $\nabla \phi, \phi, X, Y, V, W$ and $Q(0, \phi, X, Y, V, W) \equiv 0$ (which will be very important for our purpose).

Since $\phi \equiv 1=\max _{p \in M^{m}} \phi(p)$ on $F_{0}$, we see that $\nabla \phi \equiv 0$ on $F_{0}$. Hence $Q(\nabla \phi, \phi, X, Y, V, W) \equiv$ $Q(0, \phi, X, Y, V, W) \equiv 0$ and $\nabla_{V} \nabla \phi \equiv 0, \nabla_{W} \nabla \phi \equiv 0$ on $F_{0}$. Then at any point on $F_{0}$, we have

$$
\begin{gathered}
\hat{R}(X+V, Y+W, Y+W, X+V)=R(X+V, Y+W, Y+W, X+V) \\
+\frac{1}{2}[-H e s s(\phi)(X, X) g(W, W)+2 H e s s(\phi)(X, Y) g(V, W) \\
-H e s s(\phi)(Y, Y) g(V, V)] .
\end{gathered}
$$

On the other hand, let

$$
A=\left(\begin{array}{cc}
H e s s(\phi)(X, X) & \operatorname{Hess}(\phi)(X, Y) \\
\operatorname{Hess}(\phi)(X, Y) & \operatorname{Hess}(\phi)(Y, Y),
\end{array}\right), B=\left(\begin{array}{cc}
g(W, W) & -g(V, W) \\
-g(V, W) & g(V, V)
\end{array}\right) .
$$

Then

$$
\hat{R}(X+V, Y+W, Y+W, X+V)=R(X+V, Y+W, Y+W, X+V)+\frac{1}{2} \operatorname{tr}(-A B) .
$$

Since $\phi$ attains its maximum at any point on $F_{0}$, we see that $-A$ is nonnegative definite on $F_{0}$. It is easy to check that $B$ is also nonnegative definite. Hence $\operatorname{tr}(-A B) \geq 0$ (although $-A B$ is not nonnegative definite if $A B \neq B A$ ). Since $g$ has positive sectional curvature everywhere on $M^{m}$ by assumption, then at any point on $F_{0}$, we see that

$$
\hat{R}(X+V, Y+W, Y+W, X+V) \geq R(X+V, Y+W, Y+W, X+V)>0 .
$$

Hence $\hat{g}$ still has positive sectional curvature at all points on $F_{0}$.

Lemma 3.4. Let $\pi:\left(M^{m}, g\right) \rightarrow(N, h)$ be a Riemannian submersion and $g=g_{v} \oplus g_{h}$, where $g_{v} / g_{h}$ are the vertical / horizontal components of $g$, respectively. Suppose $\phi$ is a positive basic function defined on $M^{m}$. Let $\hat{g}=\left(\phi g_{v}\right) \oplus g_{h}$. Suppose $\hat{\nabla} / \nabla$ are the Levi-Civita connections and $\hat{R} / R$ are the Riemannian curvature tensors with respect to $\hat{g} / g$, respectively. Moreover, let $H e s s(\phi)$ be the Hessian of $\phi$ with respect to $g$. Then for any horizontal vector fields $X, Y$ (X,Y are not necessarily basic vector fields) and vertical vector fields $V, W$, we have

$$
\hat{\nabla}_{X} Y=\nabla_{X} Y \text {. }
$$




$$
\begin{gathered}
\hat{\nabla}_{V} W=\nabla_{V} W-\frac{g(V, W)}{2} \nabla \phi+(\phi-1)\left(\nabla_{V} W\right)^{h} . \\
\hat{\nabla}_{V} X=\nabla_{V} X+\frac{g(X, \nabla \phi)}{2 \phi} V+\frac{1-\phi}{2} \sum_{i=1}^{n} g\left(\left[X, \varepsilon_{i}\right], V\right) \varepsilon_{i} . \\
\hat{\nabla}_{X} V=\nabla_{X} V+\frac{g(X, \nabla \phi)}{2 \phi} V+\frac{1-\phi}{2} \sum_{i=1}^{n} g\left(\left[X, \varepsilon_{i}\right], V\right) \varepsilon_{i},
\end{gathered}
$$

where $\left\{\varepsilon_{i}\right\}_{i=1}^{n}$ is any orthonormal basis of the horizontal distribution with respect to $g$ and $n=$ $\operatorname{dim}(N)$.

Moreover, if $X, Y$ are basic vector fields and $V, W$ are vertical vector fields, then

$$
\begin{gathered}
\hat{R}(X+V, Y+W, Y+W, X+V)=R(X+V, Y+W, Y+W, X+V) \\
+(\phi-1) P(\nabla \phi, \phi, X, Y, V, W)+Q(\nabla \phi, \phi, X, Y, V, W) \\
+\left[-g(W, W) g\left(\nabla_{V} \nabla \phi, X\right)+g(V, W) g\left(\nabla_{W} \nabla \phi, X\right)\right. \\
\left.+g(V, W) g\left(\nabla_{V} \nabla \phi, Y\right)-g(V, V) g\left(\nabla_{W} \nabla \phi, Y\right)\right] \\
+\frac{1}{2}[-H e s s(\phi)(X, X) g(W, W)+2 H e s s(\phi)(X, Y) g(V, W) \\
-H e s s(\phi)(Y, Y) g(V, V)],
\end{gathered}
$$

where $P(\nabla \phi, \phi, X, Y, V, W), Q(\nabla \phi, \phi, X, Y, V, W)$ are two functions which depend on $\nabla \phi, \phi, X, Y, V, W$ and $Q(0, \phi, X, Y, V, W) \equiv 0$.

Proof. The proof is based on a lengthy computation and the following Koszul's formula:

$$
\begin{gathered}
2 \hat{g}\left(\hat{\nabla}_{X} Y, Z\right)=X(\hat{g}(Y, Z))+Y(\hat{g}(Z, X))-Z(\hat{g}(X, Y)) \\
+\hat{g}([X, Y], Z)-\hat{g}([Y, Z], X)-\hat{g}([X, Z], Y) .
\end{gathered}
$$

We just prove the fourth-fifth equalities in Lemma 3.4. others are left to the readers. In the computation below, we will use the following trick very often: If we encounter with anything like $\phi X$, we will rewrite $\phi X=X+(\phi-1) X$. By rewriting it in this way, we can compare new curvature terms with odd terms. We will also use the fact that $\phi$ is a basic function very often.

Now let $X, Y$ be horizontal vector fields (not necessarily basic) and $\left\{\varepsilon_{i}\right\}_{i=1}^{n}$ be any orthonormal basis of the horizontal distribution with respect to $g$. By Koszul'sformula, we see that

$$
\begin{gathered}
2 \hat{g}\left(\hat{\nabla}_{X} V, \varepsilon_{i}\right)=X\left(\hat{g}\left(V, \varepsilon_{i}\right)\right)+V\left(\hat{g}\left(\varepsilon_{i}, X\right)\right)-\varepsilon_{i}(\hat{g}(X, V)) \\
+\hat{g}\left([X, V], \varepsilon_{i}\right)-\hat{g}\left(\left[V, \varepsilon_{i}\right], X\right)-\hat{g}\left(\left[X, \varepsilon_{i}\right], V\right) \\
=V\left(\hat{g}\left(\varepsilon_{i}, X\right)\right)+\hat{g}\left([X, V], \varepsilon_{i}\right)-\hat{g}\left(\left[V, \varepsilon_{i}\right], X\right)-\hat{g}\left(\left[X, \varepsilon_{i}\right], V\right) .
\end{gathered}
$$

Since $\hat{g}_{h}=g_{h}$ and $\hat{g}_{v}=\phi g_{v}$, we get

$$
\begin{gathered}
\left.2 g\left(\hat{\nabla}_{X} V, \varepsilon_{i}\right)=V g\left(\varepsilon_{i}, X\right)\right)+g\left([X, V], \varepsilon_{i}\right)-g\left(\left[V, \varepsilon_{i}\right], X\right)-\phi g\left(\left[X, \varepsilon_{i}\right], V\right) \\
\left.=V g\left(\varepsilon_{i}, X\right)\right)+g\left([X, V], \varepsilon_{i}\right)-g\left(\left[V, \varepsilon_{i}\right], X\right)-g\left(\left[X, \varepsilon_{i}\right], V\right)+(1-\phi) g\left(\left[X, \varepsilon_{i}\right], V\right) .
\end{gathered}
$$


By Koszul's formula again, we see that

$$
\left.2 g\left(\nabla_{X} V, \varepsilon_{i}\right)=V g\left(\varepsilon_{i}, X\right)\right)+g\left([X, V], \varepsilon_{i}\right)-g\left(\left[V, \varepsilon_{i}\right], X\right)-g\left(\left[X, \varepsilon_{i}\right], V\right) .
$$

Then

$$
2 g\left(\hat{\nabla}_{X} V, \varepsilon_{i}\right)=2 g\left(\nabla_{X} V, \varepsilon_{i}\right)+(1-\phi) g\left(\left[X, \varepsilon_{i}\right], V\right) .
$$

Hence

$$
\left(\hat{\nabla}_{X} V\right)^{h}=\left(\nabla_{X} V\right)^{h}+\frac{1-\phi}{2} \sum_{i=1}^{n} g\left(\left[X, \varepsilon_{i}\right], V\right) \varepsilon_{i} .
$$

Note that $\frac{1-\phi}{2} \sum_{i=1}^{n} g\left(\left[X, \varepsilon_{i}\right], V\right) \varepsilon_{i}$ does not depend on the choice of $\left\{\varepsilon_{i}\right\}_{i=1}^{n}$. By the similar argument above, we see that

$$
\left(\hat{\nabla}_{X} V\right)^{v}=\left(\nabla_{X} V\right)^{v}+\frac{g(X, \nabla \phi)}{2 \phi} V
$$

Hence

$$
\hat{\nabla}_{X} V=\nabla_{X} V+\frac{g(X, \nabla \phi)}{2 \phi} V+\frac{1-\phi}{2} \sum_{i=1}^{n} g\left(\left[X, \varepsilon_{i}\right], V\right) \varepsilon_{i} .
$$

The similar argument will also establish the first-third equalities in Lemma 3.4. We just mention that in the proof of these equalities, the fact that $\phi$ is a basic function and hence $V \phi=0$ will be used very often.

Now we are going to prove the fifth equality in Lemma 3.4. In the following we always assume that $X, Y$ are basic vector fields. First of all, we have

$$
\begin{aligned}
& \hat{R}(X+V, Y+W, Y+W, X+V)=\hat{R}(X, Y, Y, X)+\hat{R}(V, W, W, V) \\
+ & \hat{R}(X, W, W, X)+\hat{R}(Y, V, V, Y)+2 \hat{R}(X, Y, Y, V)+2 \hat{R}(Y, X, X, W) \\
+ & \hat{R}(X, Y, W, V)+2 \hat{R}(X, W, Y, V)+2 \hat{R}(V, W, W, X)+2 \hat{R}(W, V, V, Y) .
\end{aligned}
$$

Since $\hat{g}_{h}=g_{h},\left(M^{m}, \hat{g}\right) \rightarrow(N, h)$ is still a Riemannian submersion. Then by O'Neill's formula [14], we have

$$
\begin{gathered}
I_{0}=\hat{R}(X, Y, Y, X)=R_{N}(X, Y, Y, X)-\frac{3}{4} \hat{g}\left([X, Y]^{v},[X, Y]^{v}\right) \\
=R_{N}(X, Y, Y, X)-\frac{3}{4} g\left([X, Y]^{v},[X, Y]^{v}\right)+\frac{3}{4}(1-\phi) g\left([X, Y]^{v},[X, Y]^{v}\right) \\
=R(X, Y, Y, X)+\frac{3}{4}(1-\phi) g\left([X, Y]^{v},[X, Y]^{v}\right),
\end{gathered}
$$

where $R_{N}$ is the Riemannian curvature tensor of $(N, h)$. On the other hand, by the first-fourth equalities in Lemma 3.4 .

$$
\begin{aligned}
I_{1}= & \hat{R}(V, W, W, V)=\hat{g}\left(\hat{\nabla}_{V} \hat{\nabla}_{W} W-\hat{\nabla}_{W} \hat{\nabla}_{V} W-\hat{\nabla}_{[V, W]} W, V\right) \\
= & \phi g\left(\hat{\nabla}_{V}\left[\nabla_{W} W-\frac{1}{2} g(W, W) \nabla \phi+(\phi-1)\left(\nabla_{W} W\right)^{h}\right], V\right) \\
& -\phi g\left(\hat{\nabla}_{W}\left[\nabla_{V} W-\frac{1}{2} g(V, W) \nabla \phi+(\phi-1)\left(\nabla_{V} W\right)^{h}\right], V\right)
\end{aligned}
$$




$$
\begin{gathered}
-\phi g\left(\nabla_{[V, W]} W-\frac{1}{2} g([V, W], W) \nabla \phi+(\phi-1)\left(\nabla_{[V, W]} W\right)^{h}, V\right) \\
=\phi g\left(\hat{\nabla}_{V}\left(\nabla_{W} W\right)-\hat{\nabla}_{W}\left(\nabla_{V} W\right)-\nabla_{[V, W]} W, V\right) \\
-\frac{1}{2} \phi\left[g(W, W) g\left(\hat{\nabla}_{V} \nabla \phi, V\right)-g(V, W) g\left(\hat{\nabla}_{W} \nabla \phi, V\right)\right] \\
+(\phi-1) \tilde{P}_{1}(\nabla \phi, \phi, X, Y, V, W)+\tilde{Q}_{1}(\nabla \phi, \phi, X, Y, V, W) . \\
=\phi g\left(\hat{\nabla}_{V}\left(\nabla_{W} W\right)^{v}+\hat{\nabla}_{V}\left(\nabla_{W} W\right)^{h}, V\right)-\phi g\left(\hat{\nabla}_{W}\left(\nabla_{V} W\right)^{v}+\hat{\nabla}_{W}\left(\nabla_{V} W\right)^{h}, V\right) \\
-\phi g\left(\nabla_{[V, W]} W, V\right)-\frac{1}{2} \phi\left[g(W, W) g\left(\hat{\nabla}_{V} \nabla \phi, V\right)-g(V, W) g\left(\hat{\nabla}_{W} \nabla \phi, V\right)\right] \\
+(\phi-1) \tilde{P}_{1}(\nabla \phi, \phi, X, Y, V, W)+\tilde{Q}_{1}(\nabla \phi, \phi, X, Y, V, W) .
\end{gathered}
$$

Since $\phi$ is a basic function, $g(\nabla \phi, V)=V \phi=0$. Hence $\nabla \phi$ is a horizontal vector field. Then by the first-fourth equalities in Lemma 3.4, we see that

$$
g\left(\hat{\nabla}_{W} \nabla \phi, V\right)=-g\left(\nabla_{W} V, \nabla \phi\right)+\frac{g(\nabla \phi, \nabla \phi)}{2 \phi} g(W, V)
$$

and

$$
\begin{gathered}
I_{1}=\hat{R}(V, W, W, V)=\phi R(V, W, W, V) \\
+(\phi-1) \check{P}_{1}(\nabla \phi, \phi, X, Y, V, W)+\check{Q_{1}}(\nabla \phi, \phi, X, Y, V, W) \\
=R(V, W, W, V)+(\phi-1) R(V, W, W, V) \\
+(\phi-1) \check{P}_{1}(\nabla \phi, \phi, X, Y, V, W)+\check{Q}_{1}(\nabla \phi, \phi, X, Y, V, W) \\
=R(V, W, W, V)+(\phi-1) P_{1}(\nabla \phi, \phi, X, Y, V, W)+Q_{1}(\nabla \phi, \phi, X, Y, V, W),
\end{gathered}
$$

where $P_{1}(\nabla \phi, \phi, X, Y, V, W), Q_{1}(\nabla \phi, \phi, X, Y, V, W)$ are two functions depending on $\nabla \phi, \phi, X, Y, V, W$ and $Q_{1}(0, \phi, X, Y, V, W) \equiv 0$.

Since $X$ is a basic vector field, $[X, W]$ is vertical. Hence by the first-fourth equalities in Lemma 3.4 ,

$$
\begin{gathered}
I_{2}=\hat{R}(X, W, W, X)=\hat{g}\left(\hat{\nabla}_{X} \hat{\nabla}_{W} W-\hat{\nabla}_{W} \hat{\nabla}_{X} W-\hat{\nabla}_{[X, W]} W, X\right) \\
=g\left(\hat{\nabla}_{X}\left[\nabla_{W} W-\frac{1}{2} g(W, W) \nabla \phi+(\phi-1)\left(\nabla_{W} W\right)^{h}\right], X\right) \\
-g\left(\hat{\nabla}_{W}\left[\nabla_{X} W+\frac{g(X, \nabla \phi)}{2 \phi} W+\frac{1-\phi}{2} \sum_{i=1}^{n} g\left(\left[X, \varepsilon_{i}\right], W\right) \varepsilon_{i}\right], X\right) \\
-g\left(\nabla_{[X, W]} W-\frac{1}{2} g([X, W], W) \nabla \phi+(\phi-1)\left(\nabla_{[X, W]} W\right)^{h}, X\right) \\
=R(X, W, W, X)+(\phi-1) P_{2}(\nabla \phi, \phi, X, Y, V, W) \\
+Q_{2}(\nabla \phi, \phi, X, Y, V, W)-\frac{1}{2} \operatorname{Hess}(\phi)(X, X) g(W, W),
\end{gathered}
$$

where $P_{2}(\nabla \phi, \phi, X, Y, V, W), Q_{2}(\nabla \phi, \phi, X, Y, V, W)$ are two functions depending on $\nabla \phi, \phi, X, Y, V, W$ and $Q_{2}(0, \phi, X, Y, V, W) \equiv 0$. 
By the similar argument, we see that

$$
\begin{aligned}
& I_{3}=\hat{R}(Y, V, V, Y)= R(Y, V, V, Y)+(\phi-1) P_{3}(\nabla \phi, \phi, X, Y, V, W) \\
&+Q_{3}(\nabla \phi, \phi, X, Y, V, W)-\frac{1}{2} \operatorname{Hess}(\phi)(Y, Y) g(V, V) . \\
& I_{4}=\hat{R}(X, Y, Y, V)= R(X, Y, Y, V)+(\phi-1) P_{4}(\nabla \phi, \phi, X, Y, V, W) \\
&+Q_{4}(\nabla \phi, \phi, X, Y, V, W) . \\
& I_{5}=\hat{R}(Y, X, X, W)= R(Y, X, X, W)+(\phi-1) P_{5}(\nabla \phi, \phi, X, Y, V, W) \\
&+Q_{5}(\nabla \phi, \phi, X, Y, V, W) . \\
& I_{6}=\hat{R}(X, Y, W, V)= R(X, Y, W, V)+(\phi-1) P_{6}(\nabla \phi, \phi, X, Y, V, W) \\
&+Q 6(\nabla \phi, \phi, X, Y, V, W) . \\
& I_{7}=\hat{R}(X, W, Y, V)= R(X, W, Y, V)+(\phi-1) P_{7}(\nabla \phi, \phi, X, Y, V, W) \\
&+Q_{7}(\nabla \phi, \phi, X, Y, V, W)+\frac{1}{2} H e s s(\phi)(X, Y) g(V, W) . \\
& I_{8}=\hat{R}(V, W, W, X)= \\
&+Q_{8}(\nabla \phi, \phi, X, Y, V, W)+\frac{1}{2} g(V, W) g\left(\nabla_{W} \nabla \phi, X\right)-\frac{1}{2} g(W, W) g(\nabla V \nabla \phi, X) . \\
& I_{9}=\hat{R}(W, V, V, Y)= R(W, V, V, Y)+(\phi-1) P_{9}(\nabla \phi, \phi, X, Y, V, W) \\
&+Q_{9}(\nabla \phi, \phi, X, Y, V, W)+ \frac{1}{2} g(V, W) g\left(\nabla{ }_{V} \nabla \phi, Y\right)-\frac{1}{2} g(V, V) g(\nabla W \nabla \phi, Y),
\end{aligned}
$$

where $P_{i}(\nabla \phi, \phi, X, Y, V, W), Q_{i}(\nabla \phi, \phi, X, Y, V, W)$ are two functions depending on $\nabla \phi, \phi, X, Y, V, W$ and $Q_{i}(0, \phi, X, Y, V, W) \equiv 0, i=3,4, \cdots 9$. Hence

$$
\begin{gathered}
\hat{R}(X+V, Y+W, Y+W, X+V)=I_{0}+I_{1}+I_{2}+I_{3}+2 \sum_{i=4}^{9} I_{i} \\
=R(X+V, Y+W, Y+W, X+V)+(\phi-1) P(\nabla \phi, \phi, X, Y, V, W) \\
+Q(\nabla \phi, \phi, X, Y, V, W)+\left[-g(W, W) g\left(\nabla_{V} \nabla \phi, X\right)+g(V, W) g\left(\nabla_{W} \nabla \phi, X\right)\right. \\
\left.+g(V, W) g\left(\nabla_{V} \nabla \phi, Y\right)-g(V, V) g\left(\nabla_{W} \nabla \phi, Y\right)\right] \\
+\frac{1}{2}[-H e s s(\phi)(X, X) g(W, W)+2 H e s s(\phi)(X, Y) g(V, W) \\
-H e s s(\phi)(Y, Y) g(V, V)],
\end{gathered}
$$

where $P(\nabla \phi, \phi, X, Y, V, W), Q(\nabla \phi, \phi, X, Y, V, W)$ are two functions which depend on $\nabla \phi, \phi, X, Y, V, W$ and $Q(0, \phi, X, Y, V, W) \equiv 0$.

\section{Proof of Theorem 3.1;}


Proof. We prove it by contradiction. We already proved it if $\|A\|$ is basic. Hence it suffices to show it if $H$ is basic. We prove it by contradiction. Let $\pi:\left(M^{m}, g\right) \rightarrow\left(N^{2}, h\right)$ be a Riemannian submersion such that $H$ is basic and the fibers have nonzero Euler numbers, where $\left(M^{m}, g\right)$ has positive sectional curvature and $m \geq 4$. By Lemma 3.3, there exists a metric $\hat{g}$ on $M^{m}$ such that $\pi:\left(M^{m}, \hat{g}\right) \rightarrow\left(N^{2}, h\right)$ is still a Riemannian submersion and all fibers of $\pi$ are minimal submanifolds with respect to $\hat{g}$. Furthermore, there exists some fiber $F_{0}$ such that $\hat{g}$ has positive sectional curvature at all points in $F_{0}$. Let $r$ be a fixed positive number such that the normal exponential map of $F_{0}$ is a diffeomorphism when restricted to the tubular neighborhood of $F_{0}$ with radius $r$. By continuity of sectional curvature, there exists $\epsilon, 0<\epsilon<r$ such that $\hat{g}$ has positive sectional curvature at the $\epsilon$ neighborhood of $F_{0}$. Choose another fiber $F_{1}$ such that $0<\hat{d}\left(F_{0}, F_{1}\right)<\epsilon$, where $\hat{d}\left(F_{0}, F_{1}\right)$ is the distance between $F_{0}$ and $F_{1}$ with respect to $\hat{g}$. Since $\pi:\left(M^{m}, \hat{g}\right) \rightarrow\left(N^{2}, h\right)$ is a Riemannian submersion, $F_{0}$ and $F_{1}$ are equidistant. On the other hand, since $0<\hat{d}\left(F_{0}, F_{1}\right)<\epsilon$, then for any point $q \in F_{1}$, there is a unique point $p \in F_{0}$ such that $\hat{d}(p, q)=\hat{d}\left(F_{0}, F_{1}\right)$. Let $L=\hat{d}(p, q)$ and $\gamma:[0, L] \rightarrow M^{m}, \gamma(0)=p, \gamma(L)=q$ be the unique minimal geodesic with unit speed realizing the distance between $p$ and $q$. Let $V \subseteq T_{q}\left(M^{m}\right)$ be the subspace of vectors $v=X(L)$ where $X$ is a parallel field along $\gamma$ such that $X(0) \in T_{p}\left(F_{0}\right)$. Then

$$
\begin{gathered}
\operatorname{dim}\left(V \cap T_{q}\left(F_{1}\right)\right)=\operatorname{dim}(V)+\operatorname{dim}\left(T_{q}\left(F_{1}\right)\right)-\operatorname{dim}\left(V+T_{q}\left(F_{1}\right)\right) \\
\geq(m-2)+(m-2)-(m-1)=m-3 .
\end{gathered}
$$

We claim that $\operatorname{dim}\left(V \cap T_{q}\left(F_{1}\right)\right)=m-3$. If not, then $\operatorname{dim}\left(V \cap T_{q}\left(F_{1}\right)\right)=m-2$. Let $X_{i}, i=$ $1, \cdots m-2$, be orthonormal parallel fields along $\gamma$ such that $X_{i}(0) \in T_{p}\left(F_{0}\right), X_{i}(L) \in T_{q}\left(F_{1}\right)$. For each $i$, choose a variation $f_{i}(s, t)$ of $\gamma$ such that $f_{i}(s, 0) \in F_{0}, f_{i}(s, L) \in F_{1}$ for small $s$ and $\frac{\partial f_{i}(0, t)}{\partial s}=X_{i}(t)$. By construction, $\dot{X}_{i}(t)=\hat{\nabla}_{\dot{\gamma}} X_{i}(t)=0$ for all $t$, where $\hat{\nabla}$ is the Levi-Civita connection with respect to $\hat{g}$. By the second variation formula, for $i=1, \cdots m-2$, we have

$$
\begin{gathered}
\frac{1}{2}{\frac{d^{2} E_{i}(s)}{d s^{2}} \mid s=0}_{\mid s}=\int_{0}^{L}\left(\hat{g}\left(\dot{X}_{i}, \dot{X}_{i}\right)-\hat{R}\left(X_{i}, \dot{\gamma}, \dot{\gamma}, X_{i}\right)\right) d t \\
+\hat{g}\left(\hat{B}_{1}\left(X_{i}, X_{i}\right), \dot{\gamma}\right)(L)-\hat{g}\left(\hat{B}_{0}\left(X_{i}, X_{i}\right), \dot{\gamma}\right)(0) \\
=-\int_{0}^{L} \hat{R}\left(X_{i}, \dot{\gamma}, \dot{\gamma}, X_{i}\right) d t+\hat{g}\left(\hat{B}_{1}\left(X_{i}, X_{i}\right), \dot{\gamma}\right)(L)-\hat{g}\left(\hat{B}_{0}\left(X_{i}, X_{i}\right), \dot{\gamma}\right)(0),
\end{gathered}
$$

where $E_{i}(s)=\int_{0}^{L} \hat{g}\left(\frac{\partial f_{i}(s, t)}{\partial t}, \frac{\partial f_{i}(s, t)}{\partial t}\right) d t, \hat{R}$ is the curvature tensor of $\hat{g}$ and $\hat{B}_{j}$ is the second fundamental form of $F_{j}$ with respect to $\hat{g}, j=0,1$.

Since $F_{0}$ and $F_{1}$ are minimal submanifolds in $\left(M^{m}, \hat{g}\right)$, we have

$$
\sum_{i=1}^{m-2} \hat{B}_{j}\left(X_{i}, X_{i}\right)=0, j=0,1 .
$$

Then

$$
\frac{1}{2} \sum_{i=1}^{m-2}{\frac{d^{2} E_{i}(s)}{d s^{2}}}_{\mid s=0}=-\sum_{i=1}^{m-2} \int_{0}^{L} \hat{R}\left(X_{i}, \dot{\gamma}, \dot{\gamma}, X_{i}\right) d t .
$$


Since $\hat{g}$ has positive sectional curvature at the $\epsilon$ neighborhood of $F_{0}$ and $0<\hat{d}\left(F_{0}, F_{1}\right)<\epsilon$, we see that $\hat{R}\left(X_{i}, \dot{\gamma}, \dot{\gamma}, X_{i}\right)<0$. Hence

$$
\frac{1}{2} \sum_{i=1}^{m-2}{\frac{d^{2} E_{i}(s)}{d s^{2}}}_{\mid s=0}<0 .
$$

Then there exists some $i_{0}$ such that ${\frac{d^{2} E_{i_{0}}(s)}{d s^{2}}}_{\mid s=0}<0$, which contradicts that $\gamma$ is a minimal geodesic realizing the distance between $F_{0}$ and $F_{1}$. So $\operatorname{dim}\left(V \cap T_{q}\left(F_{1}\right)\right)=m-3$. Since $\operatorname{dim}\left(T_{q}\left(F_{1}\right)\right)=m-2$, then $V \cap T_{q}\left(F_{1}\right)$ is a codimension one subspace of $T_{q}\left(F_{1}\right)$. Since $q$ is arbitrary on $F_{1}$, by doing the same construction as above for any $q$, then we get a continuous codimension one distribution on $F_{1}$. Thus the Euler number of $F_{1}$ is zero. Contradiction.

\section{Proof of theorem 1.4}

In this section we prove Theorem 1.4. Suppose $\pi:\left(M^{4}, g\right) \rightarrow\left(N^{2}, h\right)$ is a Riemannian submersion with totally geodesic fibers, where $\left(M^{4}, g\right)$ is a compact four-dimensional Einstein manifold. We are going to show that the $A$ tensor of $\pi$ vanishes and then locally $\pi$ is the projection of a metric product onto one of the factors. We firstly need the following lemmas:

Lemma 4.1. Let $\pi$ be a Riemannian submersion with totally geodesic fibers from compact Riemannian manifolds, then all fibers are isometric to each other.

Proof. See [12].

Lemma 4.2. Suppose $\pi:\left(M^{4}, g\right) \rightarrow\left(N^{2}, h\right)$ is a Riemannian submersion with totally geodesic fibers, where $\left(M^{4}, g\right)$ is a compact four-dimensional Einstein manifold. Let $c_{1}, c_{2}$ be the sectional curvature of $\left(F^{2}, g_{\mid F^{2}}\right)$ and $\left(N^{2}, h\right)$, respectively, where $g_{\mid F^{2}}$ is the restriction of $g$ to the fibers $F^{2}$. Let $\operatorname{Ric}(g)=\lambda g$ for some $\lambda$. Then

(i) $2 c_{1}+\|A\|^{2}=2 \lambda$;

(ii) $2 c_{2} \circ \pi-2\|A\|^{2}=2 \lambda$;

(iii) $\|A\|^{2}=\frac{2}{3}\left(c_{2} \circ \pi-c_{1}\right)$,

where $\|A\|^{2}=\left\|A_{X}^{*} U\right\|^{2}+\left\|A_{X}^{*} V\right\|^{2}+\left\|A_{Y}^{*} U\right\|^{2}+\left\|A_{Y}^{*} V\right\|^{2}$. Here $X, Y / U, V$ is an orthonormal basis of $\mathcal{H} / \mathcal{V}$, respectively.

Proof. See page 250, Corollary 9.62 in [3]. For completeness, we give a proof here.

Let $U, V / X, Y$ are orthonormal basis of $\mathcal{V} / \mathcal{H}$, respectively. Then by O'Neill's formula ([14]), we have

$$
\begin{gathered}
\lambda=\operatorname{Ric}(U, U)=c_{1}+\left\|A_{X}^{*} U\right\|^{2}+\left\|A_{Y}^{*} U\right\|^{2} \\
\lambda=\operatorname{Ric}(V, V)=c_{1}+\left\|A_{X}^{*} V\right\|^{2}+\left\|A_{Y}^{*} V\right\|^{2} \\
\lambda=\operatorname{Ric}(X, X)=c_{2} \circ \pi-3\left\|A_{X} Y\right\|^{2}+\left\|A_{X}^{*} U\right\|^{2}+\left\|A_{X}^{*} V\right\|^{2} \\
\lambda=\operatorname{Ric}(Y, Y)=c_{2} \circ \pi-3\left\|A_{X} Y\right\|^{2}+\left\|A_{Y}^{*} U\right\|^{2}+\left\|A_{Y}^{*} V\right\|^{2} .
\end{gathered}
$$


On the other hand, by direct calculation, we see that $2\left\|A_{X} Y\right\|^{2}=\|A\|^{2}$. Hence

$$
\begin{gathered}
2 c_{1}+\|A\|^{2}=2 \lambda ; \\
2 c_{2} \circ \pi-2\|A\|^{2}=2 \lambda ; \\
\|A\|^{2}=\frac{2}{3}\left(c_{2} \circ \pi-c_{1}\right) .
\end{gathered}
$$
$N^{2}$.

By Lemmas 4.1 and 4.2, we see that $c_{1},\|A\|$ are constants on $M^{4}$ and $c_{2}$ is a constant on

Fix $p \in M^{4}$. Locally we can always choose basic vector fields $X, Y$ such that $X, Y$ is an orthonormal basis of the horizontal distribution. At point $p$, since the image of $A_{X}^{*}$ is perpendicular to $X$ and $\operatorname{dim} \mathcal{V}=\operatorname{dim} \mathcal{H}=2, A_{X}^{*}$ must have nontrivial kernel. Then there exists some $v \in \mathcal{V}$ such that $\|v\|=1$ and $A_{X}^{*}(v)=0$. Extend $v$ to be a local unit vertical vector field $V$ and choose $U$ such that $U, V$ is a local orthonormal basis of $\mathcal{V}$.

\section{Lemma 4.3.}

$$
\begin{aligned}
& A_{X}^{*} V(p)=0 ; \\
& A_{Y}^{*} V(p)=0 .
\end{aligned}
$$

Proof. We already see $A_{X}^{*} V(p)=A_{X, p}^{*}(v)=0$. On the other hand, at point $p$, we have

$$
\begin{gathered}
A_{Y}^{*} V=g\left(A_{Y}^{*} V, X\right) X=-g\left(\nabla_{Y} V, X\right) X \\
=g\left(V, \nabla_{Y} X\right) X=g\left(V, A_{Y} X\right) X \\
=-g\left(V, A_{X} Y\right) X=-g\left(V, \nabla_{X} Y\right) X \\
=g\left(\nabla_{X} V, Y\right) X=-g\left(A_{X}^{*} V, Y\right) X=0 .
\end{gathered}
$$

Since all fibers of $\pi$ are totally geodesic, by O'Neill's formula ([14]), we see that $K(X, U)=$ $\left\|A_{X}^{*} U\right\|^{2}$. Because $\left(M^{4}, g\right)$ is Einstein, at point $p$, we have

$$
\begin{aligned}
& \lambda=\operatorname{Ric}(U, U)=c_{1}+\left\|A_{X}^{*} U\right\|^{2}+\left\|A_{Y}^{*} U\right\|^{2} ; \\
& \lambda=\operatorname{Ric}(V, V)=c_{1}+\left\|A_{X}^{*} V\right\|^{2}+\left\|A_{Y}^{*} V\right\|^{2} ;
\end{aligned}
$$

Combined with Lemma 4.3, we see that $\lambda=c_{1}$ and $\left\|A_{X}^{*} U\right\|^{2}(p)=0,\left\|A_{Y}^{*} U\right\|^{2}(p)=0$. Then $\|A\|^{2}(p)=0$. Hence $\|A\|^{2} \equiv 0$ on $M^{4}$ and $c_{1}=c_{2}$. Let $c=c_{1}=c_{2}$. Then locally $\pi$ is the projection of a metric product $B^{2}(c) \times B^{2}(c)$ onto one of the factors, where $B^{2}(c)$ is a two-dimensional compact manifold with constant curvature $c$. 


\section{Conjecture 1 and the Weak Hopf Conjecture}

In this section we point out several interesting corollaries of Conjecture 1.

Suppose $(E, g)$ is a complete, open Riemannian manifold with nonnegative sectional curvature. By a well known theorem of Cheeger and Gromoll [4], $E$ contains a compact totally geodesic submanifold $\Sigma$, called the soul, such that $E$ is diffeomorphic to the normal bundle of $\Sigma$. Let $\Sigma_{r}$ be the distance sphere to $\Sigma$ of radius $r$. Then for small $r>0$, the induced metric on $\Sigma_{r}$ has nonnegative sectional curvature by a theorem of Guijarro and Walschap [10]. In [9], Gromoll and Tapp proposed the following conjecture:

Weak Hopf Conjecture Let $k \geq 3$. Then for any complete metric with nonnegative sectional curvature on $S^{n} \times \mathbb{R}^{k}$, the induced metric on the boundary of a small metric tube about the soul can not have positive sectional curvature.

The case $n=2, k=3$ is of particular interest since the metric tube of the soul is diffeomorphic to $S^{2} \times S^{2}$.

Recall that a map between metric spaces $\sigma: X \rightarrow Y$ is a submetry if for all $x \in X$ and $r \in[0, r(x)]$ we have that $f(B(x, r))=B(f(x), r)$, where $B(p, r)$ denotes the open metric ball centered at $p$ of radius $x$ and $r(x)$ is some positive continuous function. If both $X$ and $Y$ are Riemannian manifolds, then $\sigma$ is a Riemannian submersion of class $C^{1,1}$ by a theorem of Berestovskii and Guijarro [1].

Proposition 5.1. Suppose $\Sigma$ is a soul of $(E, g)$, where $(E, g)$ is a complete, open Riemannian manifold with nonnegative sectional curvature. If the induced metric on $\Sigma_{r}$ has positive sectional curvature at some point for some $r>0$, then there is a Riemannian submersion from $\Sigma_{r}$ to $\Sigma$ with fibers $S^{l-1}$, where $l=\operatorname{dim}(E)-\operatorname{dim}(\Sigma)$.

Proof. In fact, by a theorem of Guijarro and Walschap in [11], if $\Sigma_{r}$ has positive sectional curvature at some point, the normal holonomy group of $\Sigma$ acts transitively on $\Sigma_{r}$. By Corollary 5 in [24], we get a submetry $\pi:(E, g) \rightarrow \Sigma \times[0,+\infty)$ with fibers $S^{l-1}$, where $\Sigma \times[0,+\infty)$ is endowed with the product metric. Then $\pi:\left(\pi^{-1}(\Sigma \times(0,+\infty)), g\right) \rightarrow \Sigma \times(0,+\infty)$ is also a submetry. By a theorem of Berestovskii and Guijarro in [1], $\pi$ is a $C^{1,1}$ Riemannian submersion. Then $\Sigma_{r}=\pi^{-1}(\Sigma \times\{r\})$ and $\pi: \Sigma_{r} \rightarrow \Sigma$ is also a $C^{1,1}$ Riemannian submersion with fibers $S^{l-1}$, where $\Sigma_{r}$ is endowed with the induced metric from $(E, g)$.

Proposition 5.2. When $k>n$, Conjecture 1 implies Weak Hopf Conjecture.

Proof. Suppose for some complete metric $g$ on $S^{n} \times \mathbb{R}^{k}$ with nonnegative sectional curvature, the induced metric on $\Sigma_{r}$ has positive sectional curvature for some $r>0$, where $\Sigma$ is a soul. Since $S^{n} \times \mathbb{R}^{k}$ is diffeomorphic to the normal bundle of $\Sigma$, we see that $\Sigma$ is a homotopy sphere and $\operatorname{dim}(\Sigma)=n$. By Proposition 5.1, we get a Riemannian submersion from $\Sigma_{r}$ to $\Sigma$ with fibers $S^{k-1}$, where $\Sigma_{r}$ is endowed with the induced metric from $g$ and hence has positive sectional curvature. Since $k>n$, we see $k-1 \geq n$, which is impossible if Conjecture 1 is true for $C^{1,1}$ Riemannian submersions.

Remark 3. If Remark 1 in section 3 is true, then by Proposition 5.1 again, any small metric tube about the soul can not have positive sectional curvature when the soul is odd-dimensional. This would give a solution to a question asked by K. Tapp in [19]. 


\section{References}

[1] V. N. Berestovskii and L. Guijarro, A metric characterization of Riemannian submersions. Ann. Global Anal. Geom. 18 (2000), no. 6, 577-588.

[2] M. Berger, Sur les variétés d'Einstein compactes, C. R. IIP Réunion Math. Expression Latine, Namur (1965) 35-55.

[3] A. L. Besse, Einstein Manifolds. Reprint of the 1987 edition. Classics in Mathematics. Springer-Verlag, Berlin, 2008.

[4] J. Cheeger and D. Gromoll, On the structure of complete manifolds of nonnegative curvature. Ann. of Math. 96 (1972), no. 3, 413-443.

[5] X. Chen, Curvature and Riemannian submersions. Ph.D thesis, University of Notre Dame, 2014.

[6] L. Florit and W. Ziller. Topological obstructions to fatness. Geom. Topol. 15 (2011), no. 2, 891-925.

[7] T. Frankel, Manifolds with positive curvature. Pacific J. Math. 11 (1961), 165-174.

[8] D. Gromoll and K. Grove, The low-dimensional metric foliations of Euclidean spheres. J. Differential Geom. 28 (1988), no. 1, 143-156.

[9] D. Gromoll and K. Tapp, Nonnegatively curved metrics on $S^{2} \times \mathbb{R}^{2}$. Geom. Dedicata. 99 (2003), 127-136.

[10] L. Guijarro and G. Walschap, The metric projection onto the soul. Trans. Amer. Math. Soc. 352 (2000) no. 1, 55-69.

[11] L. Guijarro and G. Walschap, The dual foliation in open manifolds with nonnegative sectional curvature. Proc. Amer. Math. Soc. 136 (2008) no. 4, 1419-1425.

[12] R. Hermann, A sufficient condition that a mapping of Riemannian manifolds be a fibre bundle. Proc. Amer. Math. Soc. 11 (1960), 236-242.

[13] N. Hitchin, Compact four-dimensional Einstein manifolds. J. Differential Geom. 9 (1974), $435-441$.

[14] B. O'Neill, The fundamental equations of a submersion. Michigan Math. J. 13 (1966), 459469.

[15] P. Petersen, Riemannian geometry. Second edition. Graduate Texts in Mathematics, 171. Springer, New York, 2006.

[16] A. Petrunin, Exercises in orthodox geometry, arXiv:0906.0290v6 [math.HO], 2013.

[17] V. Rovenski, Foliations, submanifolds, and mixed curvature. J. Math. Sci. (New York) 99 (2000), no. 6, 1699-1787.

[18] K. Tapp, Metrics with nonnegative curvature on $S^{2} \times \mathbb{R}^{4}$, Ann. Global Anal. Geom. 42 (2012), no. 1, 61-77. 
[19] K. Tapp, Rigidity for odd-dimensional souls. Geom. Topol. 16 (2012), no. 2, 957-962.

[20] P. Tondeur, Geometry of foliations. Monographs in Mathematics, 90. Birkhäuser Verlag, Basel, 1997.

[21] G. Walschap, Metric foliations and curvature. J. Geom. Anal. 2 (1992), no. 4, 373-381.

[22] B.Wilking, Index parity of closed geodesics and rigidity of Hopf fibrations. Invent. Math. 144 (2001), no. 2, 281-295.

[23] B. Wilking, Manifolds with positive sectional curvature almost everywhere. Invent Math. 148 (2002), no. 1, 117-141.

[24] B. Wilking, A duality theorem for Riemannian foliations in nonnegative sectional curvature, Geom. Funct. Anal. 17 (2007), no. 4, 1297-1320.

Department of Mathematics

University of Notre Dame

Notre Dame, Indiana, 46556.

E-mail address: xychen100@gmail.com 